\title{
Injection of recombinant tumor necrosis factor directly into liver metastases: an experimental and clinical approach
}

\author{
Jan N. M. IJzermans*, Marcel Scheringa, George P. van der Schelling, \\ Rob A. Geerling, Richard L. Marquet and Johannes Jeekel \\ Department of Surgery, Erasmus University, PO Box 1738, 3015 GD Rotterdam, \\ The Netherlands
}

(Received 14 November 1991; accepted 8 August 1991)

Systemic treatment with tumor necrosis factor (TNF) is associated with side-effects, limiting its clinical use in the treatment of malignancies. To investigate the feasibility of other routes of administration experimental and clinical studies were started to establish the toxicity and antitumor activity of TNF after intratumoral (i.t.) injection. In a rat model for colon adenocarcinoma, tumor fragments, implanted subcutaneously or under the hepatic capsule, were treated with TNF injected i.v. or i.t. A dosage of $40 \mu \mathrm{g} / \mathrm{kg}$ was lethal when given i.v., but not i.t. Injection of TNF (40 $\mu \mathrm{g} / \mathrm{kg})$ directly into the tumor resulted in inhibition of tumor growth in the subcutaneous as well as subhepatic tumor model. A phase I study was started in patients with advanced malignancies to determine the toxicity of TNF injected into liver metastases. Injection of TNF into liver metastases was accomplished by ultrasonography. A $50 \mu$ g-dose escalating schedule ( 3 patients/dosage) was chosen, starting at a dose of $100 \mu \mathrm{g}$ TNF/injection. Up to now, 12 patients have been treated, the highest dosage of TNF injected being $250 \mu \mathrm{g}$. Chills, fever, nausea and vomiting were the main side-effects. No significant changes were found in circulatory, hematologic, renal and liver parameters. In summary, i.t. administration of TNF is associated with antitumor efficacy in experimental models and well-tolerated in man. The antitumor efficacy of TNF i.t. in man awaits evaluation in a phase II study.

Keywords: liver metastases, local treatment, TNF

\section{Introduction}

Tumor necrosis factor (TNF) is a protein predominantly produced by activated macrophages $[1,2]$. Originally, TNF was named by its capacity to induce hemorrhagic necrosis of animal tumors [3]. With the availability of recombinant TNF [4] it was found that this cytokine is able to exert a broad spectrum of regulatory activities on different cell types $[5-8,29]$ and is involved in toxic and lethal effects of septicemia and endotoxic shock $[9,10]$.

It has been demonstrated in vitro that TNF is

*Corresponding author. cytotoxic to selected tumor-cell lines by interacting with specific cell surface receptors that can be induced by interferon-gamma [11]. In vivo more mechanisms may be involved in the antitumor effect of TNF, such as modulation of immune functions [1] and vascular endothelium. Recently, it was reported that TNF is able to modify the hemostatic properties of vascular endothelium in such a way that the genesis of thrombi is facilitated $[12,13]$. Furthermore, it has been demonstrated that TNF may be directly toxic to vascular endothelial cells [14]. Most likely, the sum of all these effects results in endothelial lesions of tumors 
vessels with occlusion, diminished tissue perfusion and necrosis. However, TNF not only exerts its activities on endothelial cells of newly formed vessels of tumors, but also on vascular endothelial cells of normal tissues within the whole organism. This may lead to increased vascular permeability with loss of plasma and electrolytes from vessels to the extravascular compartment and, subsequently, hypotension. Furthermore, a decrease of blood pressure may be induced by interleukin-1, a cytokine that is released by endothelial cells upon exposure to TNF $[6,15]$.

Considering the mechanism of toxicity induced by systemic TNF administration, other routes or methods of administration leading to high concentrations of this cytokine at the site of the tumor, but not in circulation, must be evaluated. We initiated the following experimental and clinical studies to investigate the toxicity and antitumor activity of intratumorally injected TNF. First, a rat model was used to study toxic and antiproliferative effects of intratumoral (i.t.) injection of recombinant TNF. Secondly, a phase I study was started in patients with therapy-resistant liver metastases to establish the toxicity of TNF injected into a tumor. The results of these experimental and clinical studies are presented.

\section{Materials and methods}

\section{Experimental study}

Rats. Male rats of the inbred WAG strain were used. The animals were bred under specific pathogen-free conditions and were 10-12 weeks old.

Tumor. A 1,2-dimethylhydrazine-induced, moderately differentiated colon adenocarcinoma (CC531), transplantable in syngeneic WAG rats, was used [7]. The tumor is weakly immunogenic as determined by the immunization challenge method of Prehn and Main [16].

TNF. Recombinant murine TNF (rMuTNF), demonstrated to be effective in the rat model, was kindly provided by $\mathrm{Dr}$ Fiers (Ghent, Belgium) [17]. The preparation used was over $99 \%$ pure, containing $72 \times 10^{6}$ units per mg protein and less than $50 \mathrm{ng}$ endotoxin per $\mathrm{mg}$ protein.

Subcutaneous tumor assay. Tumor cubes of $2 \times$ $2 \mathrm{~mm}$ were implanted in the flank of WAG rats anesthesized with ether. Growth was established by measuring with calipers the two largest perpen- dicular diameters of the subcutaneous tumor. The average diameter was taken as measure of tumor size.

Sub hepatic capsule assay (SHCA). Rats were anesthesized with ether and a median laparotomy was performed. After exposure of the liver a small incision was made in its capsule and tumor tissue of 6-8 mg was put under the capsule and pushed cephalad. After 3 weeks, rats were randomized and a second laparotomy was performed; after measuring the largest perpendicular diameters of the tumors located under the hepatic capsule either rMuTNF at a dosage of $40 \mu \mathrm{g}$ or phosphate buffered saline (PBS) at a similar volume was injected into the tumors. Eight days later, a third laparotomy was performed and tumor size was evaluated by measuring the two largest perpendicular diameters of the subcapsular tumors.

Experiments. First, toxicity and antitumor activity of rMuTNF administered by i.v. injection had to be established. Non-tumor bearing rats were treated i.v. with rMuTNF. Secondly, the effect of i.t. injected rMuTNF was established using the subcutaneous or SHCA tumor assay. rMuTNF at a dosage of $40 \mu \mathrm{g}\left(7.2 \times 10^{5} \mathrm{U} /\right.$ injection $)$ was injected into subcutaneous growing CC531 tumor parts when they had reached a size of $1 \mathrm{~cm}$ in diameter. Injection of rMuTNF was repeated 5, 10 and 15 days later. Each group contained seven animals; the experiments were performed twice. Three weeks after tumor implantation in the SHCA assay a single dose $(40 \mu \mathrm{g})$ of rMuTNF was injected into the subcapsular growing tumors. Experimental and control groups contained seven animals.

Statistical analysis. Results were analysed using Student's $t$ and Wilcoxon's rank sum test. A $P$-value less than 0.05 was considered to indicate a significant difference between parameters.

\section{Clinical study}

Patients. Informed consent was obtained from 12 adult patients (four female and eight male). The study protocol had been approved by the institutional ethics committee. Criteria for entry into the study included histologically proven liver metastases refractory to standard anticancer therapy with normal renal and hematologic functions. Increase of bilirubin and alkaline phosphatase were accepted up to two times normal and not due to common bile duct obstruction. Life expectancy had 
to be more than 3 months, with a performance status of $0-2$; former chemotherapy, radiotherapy or surgery had to be more than 4 weeks ago. Exclusion criteria were formed by hemorrhagic disease, cardiac disease and abnormal neurologic examination. At entry of this study, patients were evaluated by medical history, physical examination, performance status, coagulation profile, complete blood cell count, determination of liver renal function, and determination of serum electrolytes.

$r T N F$. Recombinant human TNF (rTNF; provided by Knoll, Ludwigshafen, Germany) had a specific activity of $6.63 \times 10^{6} \mathrm{U} / \mathrm{mg}$ protein. The preparation used was more than $99 \%$ pure and contained less than $10 \mathrm{pg}$ endotoxin per $\mathrm{mg}$ protein.

Experimental design. The phase I protocol included a single i.t. injection of rTNF into a liver metastasis by ultrasound guidance. To assess toxicity, rTNF was administered according to a $50 \mu \mathrm{g}$ escalating dose schedule with three patients at each dose level. If a patient experienced no serious toxic side-effects (defined according to the WHO criteria as less than grade 2) the protocol permitted administration of a second, dose escalating TNF injection to the same patient after a period of at least 4 weeks. The study was started at a dosage of $100 \mu \mathrm{g}$ rTNF/injection. All patients received Indomethacin $(50 \mathrm{mg} / \mathrm{supp}) 30 \mathrm{~min}$ before rTNF injection. Laboratory data were obtained before and $24-72 \mathrm{~h}$ after rTNF injection. Vital signs (pulse, blood pressure and temperature) were monitored hourly during the first day after injection and repeated three times daily until the fifth day after puncture. Two patients were treated twice at different dosages after an interval of 4 weeks.

Statistics. Data before and after TNF administration were compared using the paired Student's

Table 1. Mortality due to rMuTNF treatment of rats

\begin{tabular}{lll}
\hline $\begin{array}{l}\text { TNF-dose } \\
(\mu \mathrm{g} / \mathrm{kg})\end{array}$ & Route of administration & Mortality \\
\hline 4 & i.v.; daily & $0 / 6$ \\
8 & i.v.; daily & $6 / 6$ \\
8 & i.v.; every other day & $1 / 6$ \\
40 & i.v.; single injection & $6 / 6$ \\
40 & i.v.; single + Indomethacin & $0 / 6$ \\
40 & i.t.; weekly & $0 / 6$ \\
\hline
\end{tabular}

i.t. = intratumoral; i.v. = intravenous. $t$-test. A $P$-value less than 0.05 was considered to indicate a significant difference.

\section{Results}

\section{Experimental study}

A single i.v. injection of rMuTNF at a dosage of $40 \mu \mathrm{g} / \mathrm{kg}$ body weight was uniformly lethal to all animals within $3-5 \mathrm{~h}$ (Table 1 ). As reported earlier, animals treated with rMuTNF at this dosage became diarrheaic within $1 \mathrm{~h}$ and had manifest blood loss in their feces [18]. At autopsy a hemorrhagic colitis was found that was mainly restricted to the caecum. No other signs of gastro-intestinal inflammation were found. A single i.v. injection of rMuTNF at a dosage of $8 \mu \mathrm{g} / \mathrm{kg}$ body weight was well-tolerated by all animals and could be repeated on alternate days without leading to hemorrhagic colitis. Dosages of $4 \mu \mathrm{g} / \mathrm{kg}$ could be given daily.

Pretreatment of the animals with Indomethacin (i.p.; $10 \mathrm{mg} / \mathrm{kg}$ body weight, $1 \mathrm{~h}$ before TNF) could abolish the lethal effect of i.v. injection of rMuTNF at a dosage of $40 \mu \mathrm{g} / \mathrm{kg}$. Animals treated in this way only developed mild transient diarrhea. This combined therapy could be repeated three times on alternate days before signs of hemorrhagic colitis were observed by blood loss in feces. However, this rMuTNF treatment did lead to excessive wasting of up to $25 \%$ of the body weight within 1 week.

In the subcutaneous assay i.t. injection of rMuTNF at a dosage of $40 \mu \mathrm{g} / \mathrm{kg}$ was well-tolerated. None of the animals treated with i.t. injection of rMuTNF at a dosage of $40 \mu \mathrm{g} / \mathrm{kg}$ showed

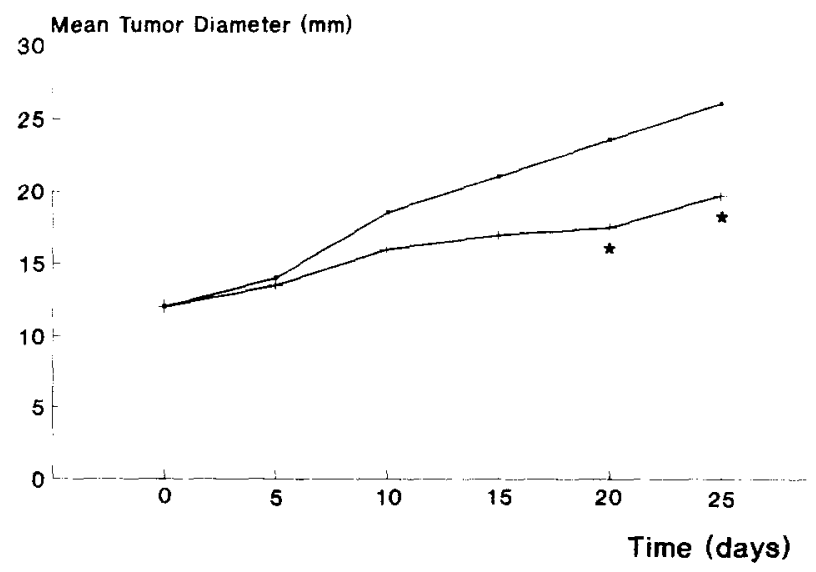

Figure 1. Antitumor activity (+) of rMuTNF injected into subcutaneous CC531 tumors, compared with controls $(-)$; ${ }^{*} P<0.05$ according to Wilcoxon's rank sum test. 
blood loss in their feces or developed diarrhea, nor had excessive weight loss.

The antitumor effect of rMuTNF after injection into subcutaneous growing CC531 tumors on days $0,5,10$ and 15 is shown in Figure 1. It was found that rMuTNF inhibited tumor growth significantly. At day 25 control tumors had a mean diameter of $26.0 \mathrm{~mm}$, while rMuTNF-treated tumors showed a mean diameter of $19.7 \mathrm{~mm}$. However, the difference between both groups was mainly due to specific types of necrotic lesions observed in only a few of the experimental animals. Not all tumors treated with rMuTNF showed the same response to treatment. Two of the rMuTNF-treated tumors showed hemorrhagic necrosis while others did not. In the control group such lesions were never observed after i.t. injection of PBS. In the SHCA assay i.t. administration of rMuTNF resulted in a significant tumor growth inhibition (Table 2). Eight days after i.t. injection of PBS, respectively rMuTNF, five out of seven control tumors had progressed compared with only one out of six rMuTNF-treated tumors. rMuTNF-treated tumors showed significantly more necrotic lesions than control tumors. None of the i.t. injected rats had

Table 2. Antitumor activity of rMuTNF injected at a dosage of $40 \mu \mathrm{g}$ into CC531 tumors implanted under the hepatic capsule

\begin{tabular}{ll}
\hline & Tumor growth (mm) \\
\hline rMuTNF & $-5,-1,0,0,0,1$ \\
PBS & $-5,-1,1,3,5,6,6$ \\
\hline
\end{tabular}

According to Wilcoxon's rank sum test $P<0.05$.

Table 3. Characteristics of patients treated with rTNF intratumorally

\begin{tabular}{llll}
\hline Patient & Dose & Diagnosis & Sex/age \\
\hline 1 & 100 & Colon carcinoma & $\mathrm{F} / 54$ \\
2 & 100 & Pancreatic carcinoma & $\mathrm{M} / 62$ \\
3 & 100 & Adenocarcinoma & $\mathrm{F} / 45^{*}$ \\
4 & 150 & Adenocarcinoma & $\mathrm{F} / 45$ \\
5 & 150 & Pancreatic carcinoma & $\mathrm{M} / 46$ \\
6 & 150 & Bile duct carcinoma & $\mathrm{F} / 44^{*}$ \\
7 & 200 & Colon carcinoma & $\mathrm{M} / 46$ \\
8 & 200 & Colon carcinoma & $\mathrm{M} / 52$ \\
9 & 200 & Stomach carcinoma & $\mathrm{M} / 72$ \\
10 & 250 & Bile duct carcinoma & $\mathrm{F} / 44$ \\
11 & 250 & Colon carcinoma & $\mathrm{M} / 46$ \\
12 & 250 & Colon carcinoma & $\mathrm{F} / 54$ \\
\hline
\end{tabular}

* Patients treated with escalating dosages of rTNF. signs of rMuTNF toxicity. One of the experimental rats died during the second laparotomy due to ether toxicity.

\section{Clinical study}

Characteristics of the patients studied are given in Table 3; the side-effects observed after rTNF injection in Table 4. Nine out of 10 patients became febrile, with temperature peaks rising to $39.8^{\circ} \mathrm{C}$ within 1-2 h after i.t. injection of rTNF. Body temperature returned to normal values within 3-4 h. In most patients fever was preceded by rigors that were not prevented with Indomethacin. Two patients pretreated with Indomethacin developed mild chills without fever. Generally, rigors started 10-20 min after i.t. injection of rTNF and lasted for about $30 \mathrm{~min}$. No relationship was found between the dose of rTNF given and the intensity of systemic side-effects, including rigors, fever and nausea. A fall in blood pressure (not $<90 \mathrm{mmHg}$ ) requiring volume substitution was observed in one patient treated concomitantly with morphine. No dose-effect relation was observed between rTNF and blood pressure. The patients studied had no significant changes of hematologic parameters. Intratumoral injections of rTNF neither had an effect on renal function or electrolytes, including sodium and potassium, nor on white blood cell count and differential. In addition, liver function tests showed no significant changes in bilirubin, alkaline phosphatase, SGOT, SGPT, $\gamma$-GT and LDH. At follow-up examinations $24-48 \mathrm{~h}$ after rTNF injection all laboratory parameters were found unchanged when compared with pretreatment values. No patient showed a significant weight loss.

An antitumor effect of rTNF, as measured by sonographic control, was not observed in any of

Table 4. Toxicity of rTNF injected into liver metastases

\begin{tabular}{lllll}
\hline $\begin{array}{l}\text { Dose }\left(10^{6} \mathrm{U}\right) / \text { injection } \\
\mu \mathrm{g} / \text { injection }\end{array}$ & 0.6 & 0.9 & 1.3 & 1.7 \\
& 100 & 150 & 200 & 250 \\
\hline Fever $\left(>38^{\circ} \mathrm{C}\right)$ & $3 / 3$ & $2 / 3$ & $3 / 3$ & $1 / 3$ \\
Chills & $2 / 3$ & $3 / 3$ & $3 / 3$ & $3 / 3$ \\
Nausea & $2 / 3$ & $2 / 3$ & $3 / 3$ & $1 / 3$ \\
Vomiting & $1 / 3$ & $2 / 3$ & $1 / 3$ & $1 / 3$ \\
Diarrhea & $0 / 3$ & $0 / 3$ & $0 / 3$ & $0 / 3$ \\
Hypotension $\left(<90\right.$ syst $^{*}$ & $0 / 3$ & $0 / 3$ & $0 / 3$ & $0 / 3$ \\
Abnormal liver function & $0 / 3$ & $0 / 3$ & $0 / 3$ & $0 / 3$ \\
Leukopenia $\left(<3000 / \mathrm{mm}^{3}\right)$ & $0 / 3$ & $0 / 3$ & $0 / 3$ & $0 / 3$ \\
Thrombocytopenia $\left(<10^{5} / \mathrm{mm}^{3}\right)$ & $0 / 3$ & $0 / 3$ & $0 / 3$ & $0 / 3$ \\
\hline
\end{tabular}

*Changes in liver enzymes: bilirubin, alkaline phosphatase, SGOT, SGPT, LDH, gamma-GT. 
the patients treated. However, it should be stressed that this study is a phase I trial, intended to evaluate the toxicity of rTNF. The diameter of the liver metastases treated showed a wide range of $2-9 \mathrm{~cm}$, which limits the evaluation of antitumor responses in these patients.

\section{Discussion}

In the experimental part of this study rMuTNF was found to exert anti tumor activity against a rat colon carcinoma when administered i.v. as well as directly into the tumor. These results are in agreement with those of others $[19,20]$. In vitro experiments of the CC531 cell line showed no sensitivity for rMuTNF. These findings led to the suggestion that indirect mechanisms are responsible for the antitumor effects seen in vivo, such as activation of immunocompetent cells [21]. The immune system seemed to be also important with regard to rMuTNF treatment in our SHCA experiments. When animals were treated with cyclosporin A prior to and during treatment, rMuTNF had only half the growth inhibiting effects as without cyclosporin treatment (unpublished results).

The toxic effects of rMuTNF were impressive. High dosages of rMuTNF led to death of normal WAG rats within several hours. The most striking finding at autopsy was a severe hemorrhagic colitis. The colon and especially the cecum appeared to be very sensitive to the toxic effects of rMuTNF, and massive necrosis was found in this part of the intestinal tract. Remarkably, the small intestine was not involved.

Of main interest for the aim of our study was the observation that dosages of rMuTNF that appeared to be lethal when administered i.v., were well-accepted when injected directly into the tumor. Hemorrhagic colitis, one of the most serious side-effects after i.v. injection of rMuTNF, was not observed in any of the animals treated by i.t. injection of rMuTNF at high dosages. Furthermore, no site-dependent toxicity was found. As in the subcutaneous tumor assay rTNF could be injected in tumors located in the liver without inducing toxic side-effects.

The in vitro and in vivo results with TNF gave high expectations for the clinical use in cancer patients. However, the clinical results with systemic application of rTNF are disappointing: toxicity is high and only sporadic tumor responses are seen. We conducted this phase I trial to achieve maximal local concentrations of rTNF at the tumor site and at the same time reduce systemic toxicity. In the clinical study the feasibility of i.t. injection of rTNF into liver metastases was demonstrated. The main clinical side-effects were fever and rigors. Whether these symptoms were caused by rTNF itself or other cytokines released by TNF, such as interleukin-1 [6], remains to be determined. It should be noted that the same flu-like symptoms have been reported in studies investigating the effects of other cytokines, such as interleukin-2 [22] and interferon- $\gamma$ [23]. This finding may indicate a common pathway responsible for these side-effects. In the current study no dose-response effect was found with regard to toxicity. Fall in blood pressure was observed in one patient treated concomitantly with morphine. As both agents can induce a decrease of blood pressure and may act synergistically, concomitant use should be avoided. One patient received an anti-emeticum. Besides these two patients other patients were treated only with Indomethacin and rTNF. Further evaluation of the escalating dose schedule will reveal the dose-limiting side-effect of rTNF injected intratumorally into liver metastases.

Pretreatment with Indomethacin did not completely prevent rTNF toxicity, although pretreatment with this NSAID certainly may have reduced side-effects.

In this study rTNF was injected intratumorally into liver metastases to minimize systemic sideeffects. The preliminary results suggest that this route of administration of rTNF into liver metastases is well-accepted and does not lead to serious toxicity as in systemic treatment with rTNF. In studies using systemic application of rTNF at dosages limited by toxicity, antitumor responses were seldom observed [24, 25]. In contrast, studies of local application of rTNF reported comparable or reduced toxicity [26,27]. Although these were phase I trials and therefore no conclusions can be drawn on the effectiveness of i.t. injection of rTNF, regression of tumor growth was seen. A phase II trial of intratumoral application of rTNF in patients with Kaposi's sarcoma showed tumor regression in 15 of the 16 tumors injected [28]. These findings suggest that the local concentration of TNF at the tumor site is of utmost importance, while circulating systemic TNF mainly stimulates mechanisms leading to side-effects but not playing a pivotal role in antitumor activity. Thus, the i.t. injection of rTNF into liver metastases, alone or in combination with other anticancer regimens may offer new perspectives to evaluate the potency of TNF in cancer treatment. 


\section{References}

1. Balkwill FR, 1989, Tumour necrosis factor. British Medical Bulletin, 45, 389-400.

2. Männel DN, Moore $\mathrm{RN}$ and Mergenhagen SE, 1980, Macrophages as a source of tumoricidal activity (tumor-necrotizing factor). Infections and Immunity, 30, 523-530.

3. O'Malley WE, Achinstein B and Shear MH, 1962, Action of bacterial polysaccharide on tumours II. Damage of sarcoma 37 by serum of mice treated with Serratia marcescens polysaccharide, and induced tolerance. $J N C l, 29,1169-1175$.

4. Pennica D, Nedwin GE, Hayflick JS, Seeburg PH, Derynck R, Palladino MA, Kohr WJ, Aggarwal BB and Goeddel DV, 1984, Human tumour necrosis factor: precursor structure, expression and homology to lymphotoxin. Nature, 312, 724-729.

5. Collins T, Lapierre LA, Fiers W, Strominger JL and Pober JS, 1986, Recombinant human tumor necrosis factor increases mRNA levels and surface expression of HLA-A, B antigens in vascular endothelial cells and dermal fibroblasts in vitro. Proceedings of the National Academy of Sciences USA, 83, 446-450.

6. Dinarello CA, Cannon JG, Wolff SM, Bernheim HA, Beutler B, Cerami A, Figari IS, Palladino MA $\mathrm{Jr}$ and O'Connor JV, 1986, Tumor necrosis factor (cachectin) is an endogenous pyrogen and induces production of interleukin-1. Journal of Experimental Medicine, 163, 1433-1450.

7. Marquet RL, Westbroek DL and Jeekel J, 1984, Interferon treatment of a transplantable colon adenocarcinoma; importance of tumor site. International Journal of Cancer, 33, 689-692.

8. Torti FM, Dieckmann B, Beutler B, Cerami A and Ringold GM, 1985, A macrophage factor inhibits adipocyte gene expression: an in vitro model of cachexia. Science, 229, 867-869.

9. Beutler BA and Cerami AC, 1986, Cachectin and tumour necrosis factor as two sides of the same biological coin. Nature, 320, 584-588.

10. Tracey KJ, Lowry SF and Fahey TJ, 1987, Cachectin/tumor necrosis factor induces lethal shock and stress hormone responses in the dog. Surgical Gynecology and Obstetrics, 164, 415-421.

11. Aggarwal BB, Eessalu TE and Hass PE, 1985, Characterization of receptors for human tumour necrosis factor and their regulation by gamma interferon. Nature, 318, 665-666.

12. Bevilacqua MP, Pober JS, Majeau GR, Fiers W, Cotran RS and Gimbrone MA, Jr, 1986, Recombinant tumor necrosis factor induces procoagulant activity in cultured human vascular endothelium: characterization and comparison with the actions of interleukin-1. Proceedings of the National Academy of Sciences USA, 83, 4533-4537.

13. Nawroth PP and Stern DM, 1986, Modulation of endothelial cell hemostatic properties by tumor necrosis factor. Journal of Experimental Methods, 163, $740-745$.

14. Sato N, Goto T, Harnaka K, Satomi N, Nariuchi H,
Mano-Hirano Y and Sawasaki Y, 1986, Actions of tumor necrosis factor on cultured vascular endothelial cells: morphologic modulation, growth inhibition, and cytotoxicity. INCI, 76, 1113-1121.

15. Nawroth PP, Bank I, Handley D, Cassimeris J, Chess L and Stern D, 1986, Tumor necrosis factor/ cachectin interacts with endothelial cell receptors to induce release of interleukin-1. Journal of Experimental Methods, 163, 1363-1375.

16. Prehn RT and Main JW, 1957, Immunity to methylcholanthrene induced sarcomas. Journal of the $\mathrm{Na}$ tional Cancer Institute, 18, 769-778.

17. Fransen L, Muller R, Marmenout A, Tavenier J, van der Heyden J, Kawashima E, Chollet A, Tizard $R$, van Heuverswyn $H$, van Vliet $A$, Ruysschaert $M$ and Fiers W, 1985, Molecular cloning of mouse tumor necrosis factor cDNA and its eukaryotic expression. Nucleic Acids Research, 13, 4417-4429.

18. Marquet RL, IJzermans JNM, de Bruin RWF, Fiers W and Jeekel J, 1987, Antitumor activity of recombinant mouse tumor necrosis factor (TNF) on colon cancer in rats is promoted by recombinant rat interferon-gamma; toxicity is reduced by indomethacine. International Journal of Cancer, 40, 550-553.

19. Fiers W, Brouckeart P, Guiser Y, Remaut E, vanRoy F, Devos R, Fransen L, Leroux-Roels G, Marmenout A, Tavernier $\mathbf{J}$ and van der Heyden $\mathbf{J}$. Recombinant interferon gamma and its synergism with tumor necrosis factor in the human and mouse systems. In: Stewart WE and Schellekens H, eds. The Biology of the Interferon System. London: Elsevier Science Publishers, 1986, pp. 112-120.

20. Haranaka K, Satomi N and Sakurai A, 1984, Antitumor activity of murine tumor necrosis factor (TNF) against transplanted murine tumors and heterotransplanted human tumors in nude mice. International Journal of Cancer, 34, 263-267.

21. Havell EA, Fiers $W$ and North RJ, 1988, The antitumor function of tumor necrosis factor (TNF) $I$. Journal of Experimental Methods, 167, 1067-1085.

22. Rosenberg SA, 1986, The adoptive immunotherapy of cancer using the transfer of activated lymphoid cells and interleukin-2. Seminars in Oncology, 13, 200-206.

23. Vadhan-raj S, Nathan C, Bhalla R, Pelus L, Nathan CF, Sherwin SA, Oettgen HF and Krown SE, 1986, Phase I trial of recombinant interferon gamma by six hours intravenous infusion. J. Clin. Oncol, 4, 137-146.

24. Creaven PJ, Benner DE, Cowens JW, Huben RP, Wof RM, Takita H, Arbuck SG, Razack MS and Proefrock AD, 1989, A Phase I clincal trial of recombinant tumor necrosis factor given daily for five days. Cancer Chemotherapy and Pharmacology, 23, 186-189.

25. Steinmetz T, Schaadt M, Gahl R, Schenk V, Diehl $\mathrm{V}$ and Pfreundschuh M, 1988, Phase I study of 24-hour continuous infusion of recombinant human tumor necrosis factor. Journal of Biological Response Modifiers, 7, 417-423.

26. Bartsch HH, Pfizenmaier K, Schroeder M and Nagel 
GA, 1989, Intralesional application of recombinant human tumor necrosis factor alpha induces local tumor regression in patients with advanced malignancies. European Journal of Cancer and Clinical Oncology, 25, 287-291.

27. Pfreundschuh MG, Steinmetz HT, Tueschen R, Schenk V, Diehl V and Schaadt M, 1989, Phase I study of intratumoral application of recombinant human tumor necrosis factor. European Journal of Cancer and Clinical Oncology, 25, 379-388.
28. Kahn JO, Kaplan LD, Volberding PA, Ziegler JL, Crowe S, Saks SR and Abrams DI, 1989, Intralesional recombinant tumor necrosis factor-alpha for AIDS-associated Kaposi's sarcoma: a randomized, double-blind trial. Journal of Acquired Immune Deficiency Syndrome, 2, 217-223.

29. Munker R, Gasson J, Ogawa M and Koeffler HP, 1986, Recombinant human TNF induces production of granulocyte-monocyte colony-stimulating factor. Nature, 323, 79-82. 